\title{
Frekans Modüleli Sürekli Dalga Radarıyla Simüle Edilen Hayati Sinyallerin Temassız Tespiti
}

\author{
İbrahim Şeflek ${ }^{1 *}$, Ercan Yaldız ${ }^{1}$ \\ ${ }^{1}$ Konya Teknik Üniversitesi, Mühendislik ve Doğa Bilimleri Fakültesi, Elektrik-Elektronik Mühendisliği Bölümü, Konya, Türkiye (ORCID: 0000-0002-6782-9513) \\ ${ }^{1}$ Konya Teknik Üniversitesi, Mühendislik ve Doğa Bilimleri Fakültesi, Elektrik-Elektronik Mühendisliği Bölümü, Konya, Türkiye (ORCID: 0000-0003-2245-0654)
}

( $1^{\text {st }}$ International Conference on Computer, Electrical and Electronic Sciences ICCEES 2020 - 8-10 Ekim 2020)

(DOI: $10.31590 /$ ejosat.802905)

ATIF/REFERENCE: Şeflek, İ. \& Yaldız, E. (2020). Frekans Modüleli Sürekli Dalga Radarıyla Simüle Edilen Hayati Sinyallerin Temassız Tespiti. Avrupa Bilim ve Teknoloji Dergisi, (Özel Sayı), 72-77.

$\ddot{O} \mathbf{z}$

Radarların son dönemde iç ortam uygulamalarında da kullanılabilmeleri onları hayatın bir parçası haline getirmiştir. Yaşam alanının herhangi bir yerinde (ev, araç, sokak) radarlara rastlama olasılığı oldukça artmıştır. Bu artışa paralel olarak hayatı kolaylaştıran radar uygulamaları da ortaya çıkmıştır. Özellikle radarların bir nesnenin varlığını temassız tespit edebilme yeteneği onların tıp uygulamalarında da yerini bulmasını sağlamıştır. Hayati belirtilerin radarlarla temassız tespiti son dönemlerde dikkat çeken çalışmalar arasında yerini almaktadır. Bu tespitte hasta için temaslı herhangi bir durum (prob vb.) söz konusu olmadığı için hareket serbestliği sağlamakta ayrıca gözetimi gerçekleştirecekler için işin kolaylaşmasına sebep olmaktadır. Frekans Modüleli Sürekli Dalga Radarının (FMCW) basit yapısı, ucuz maliyeti, menzil tespit edebilme yeteneği ve darbe radarlarına kıyasla gürültüden daha az etkilenmesi onu diğer radarlardan bir adım öne çıkarmaktadır. Ayrıca sürekli dalga radarlarının (CW) birden çok hedefi tespit edememe dezavantajı FMCW radar ile ortadan kaldırılmaktadır. FMCW radar menzil çözünürlüğüne bağlı olarak birden çok hedefi birbirinden ayırt edebilmektedir. Bu çalışmada, solunum ve kalp atışından kaynaklanan göğüs duvar hareketi simüle edilerek FMCW radarı tarafından tespiti gerçekleştirilmiştir. Tespitin gerçekleştirilebilmesi için bir algoritma sunulmuştur. Öncelikli olarak hedeflerin menzili tespit edilmiş daha sonra hayati sinyaller hesaplanmıştır. Çoklu sabit hedefler kullanılarak 5 ve 8 metre de bulunan hedefler sırasıyla \%0 ve \%1.56 hata oranları ile tespit edilmiştir. Bu hedeflerde mevcut olan solunum ve kalp atış hızları her iki hedef için sırasıyla \%2.29 hata oranı ile hesaplanmıştır. Elde edilen ön sonuçlar FMCW radarın menzil ve yer değiştirme kaynaklı hayati sinyal varlığının tespitinde yüksek başarıya sahip olduğunu göstermektedir. Gelecekte temaslı ölçümlerle gerçekleştirilen uygulamaların yerini radarların alabileceği öngörülmektedir.

Anahtar Kelimeler: Frekans Modüleli Sürekli Dalga Radarı, Menzil tespiti, Hayati sinyal tespiti, Temassız ölçüm, Simülasyon.

\section{Non-Contact Detection of Simulated Vital Signs with Frequency Modulated Continuous Wave Radar}

\begin{abstract}
The recent use of radars in indoor applications has made them a part of live. The probability of seeing radars anywhere in living area (house, car, street) has increased considerably. In parallel with this increase, radar applications that make life easier have emerged. In particular, the ability of radars to detect the presence of an object without contact has enabled them to find their place in medical
\end{abstract}

* Sorumlu Yazar: Konya Teknik Üniversitesi, Mühendislik ve Doğa Bilimleri Fakültesi, Elektrik-Elektronik Mühendisliği Bölümü, Konya, ORCID:0000-0002-6782-9513, iseflek@ktun.edu.tr 
applications. Non-contact detection of vital signs with radars is among the remarkable studies recently. Since there is no contact situation (probe, etc.) for the patient in this determination, it provides freedom of movement and also facilitates the work for those who will perform the surveillance. The simple structure of the Frequency Modulated Continuous Wave Radar (FMCW), its low cost, its ability to detect range and its less susceptibility to noise compared to pulse radars make it one step ahead of other radars. In addition, the disadvantage of continuous wave radars $(\mathrm{CW})$ that cannot detect more than one target is eliminated by FMCW radar. FMCW can distinguish multiple targets from each other, depending on the radar range resolution. In this study, chest wall motion due to respiration and heartbeat was simulated and detected by FMCW radar. An algorithm has been presented to make the detection. First of all, the range of the targets is determined and then the vital signs are calculated. By using multiple stationary targets, targets at 5 and 8 meters are detected with $0 \%$ and $1.56 \%$ error rates, respectively. Respiration and heart rates present at these targets are determined with an error rate of $2.29 \%$ for both targets, respectively. The preliminary results show that the FMCW radar has high success in detecting range and the existence of vital signs originating from displacement. It is anticipated that in the future, applications carried out with contact measurements will be replaced by radars.

Keywords: Frequency Modulated Continuous Wave Radar, Range detection, Vital signal detection, Non-contact measurement, Simulation.

\section{Giriş}

Bir nesnenin temassız uzaktan algılanması yaklaşık doksan yıldır bilim insanlarının araştırma konuları arasında yerini almaktadır. $\mathrm{Bu}$ zaman dilimi içerisinde teknolojinin gelişmesiyle birçok cihaz vasıtasıyla temassız uzaktan algılama gerçekleştirilebilmektedir. Kameralar, lidarlar ve radarlar bu algılamayı sağlamak için en yoğun kullanılan cihazlar olmaktadır. Bunların avantaj ve dezavantajları söz konusudur. Işık miktarı, nem, sis vb. çevresel faktörler cihazların performansını oldukça etkilemektedir. Bütün bu faktörler göz önüne alındığında elektromanyetik dalgaları kullanan radarların çalışma ortamına bağımlılığının oldukça düşük olması, titreşimlere karşı yüksek hassasiyet göstermesi, nüfuz etme özellikleri ve son dönemde maliyetinin düşük olması onu kullanılabilirlik açısından diğerlerine göre ön plana çıkarmaktadır [1].

Daha önceleri büyük ve hantal yapıda olan ve dış ortamda kullanım içim tasarlanan radarlar boyutlarının küçülmesi sonucu iç ortamda evlerin içinde rahatlıkla kullanılabilen cihazlar haline gelmektedir [2]. Bu durum onların tıbbi uygulamalar için kullanılabilme kabiliyetini gün yüzüne çıkarmaktadır. Özellikle solunum ve kalp atışından kaynaklı göğüs duvarının yer değiştirme hareketi; titreşimlere karşı yüksek hassasiyet gösteren radarların bu fizyolojik parametrelerin tespitini sağlayarak insanlarda hem solunum hem de dolaşım sistemlerinin durumunu doğrudan yansıtabilmektedir. Bu nedenle, hayati belirtilerin temassız tespiti veya izlenmesi için çok uygun olabilirler. Vücuda temas edilerek bu parametrelerin eldesini sağlayan geleneksel yöntemlerin aksine radarların kullanımı hastalar için daha fazla rahatlık ve sağlık çalışanları adına ek iş yükü olmadan sürekli ve zamanında solunum ve kalp hızı izleme avantajlarına sahiptir [3]. Şiddetli yanık veya bulaşıcı hastalık, uyku apnesi izleme, ani bebek ölüm sendromu izleme yaşlı ev sağlığı ve psikolojisi gibi durumlar için radarların kullanılabilirliği çalışmalarla gösterilmiştir [4]-[9].

Sürekli dalga $(\mathrm{CW})$ radarları hayati sinyallerin tespiti ve dolayısıyla buna bağlı durumların ortaya çıkarılması için geleneksel olarak kullanılan en temel radar tipidir [10]-[12]. Düşük maliyeti ve basit devre yapısı tercih edilme sebeplerindendir. Ancak DC offset, çok yollu yansıma ve en önemlisi menzil tespitinin çalışma ortamındaki tüm nesnelerdeki bütün yansımaları tek bir sinüzoid sinyalinde toplamasından dolayı gerçekleştirilememesidir. Bunlar CW radarların dezavantajlarını oluşturmaktadır [3]. Temassız hayati sinyal izlemede menzilin de elde edilebilmesi ve CW radarların dezavantajlarını ortadan kaldırmak amacıyla ultra geniş bant (UWB) darbe radarları ve frekans modüleli sürekli dalga (FMCW) radarlar kullanılmaktadır. UWB darbe radarları yüksek uzaysal çözünürlük elde eder ancak darbe genişliği ve sinyal yoğunluğu bu radarları sınırlandırmakta ve maliyetini artırmaktadır [13]. FMCW radar, UWB radarların menzil tespit edebilme kapasitesine ek olarak CW Doppler radarının hassasiyeti ve sağlamlığını barındırmaktadır. Bu özellikler FMCW radarı oldukça kullanışlı hale getirmektedir. Buna ek olarak, FMCW radar küçük boyuta sahip olma, hafif olma, düşük güç tüketme özelliklerine sahiptir. FMCW radarın temel avantajı farklı mesafelerdeki yansımaları ayırabilmesi ve göğüs duvarındaki salınımlar nedeniyle farklı menzillerde bulunan çoklu hedeflerin saptanması ve izlenmesinde kullanılabilir olmasıdır [14]-[16]. Bu nedenle, hastane ve evde bakım gibi ortamlarda kullanılabilirliği mümkündür.

\section{Materyal ve Metot}

Bir FMCW radarın temel blok diyagramı Şekil-1'de görülmektedir.

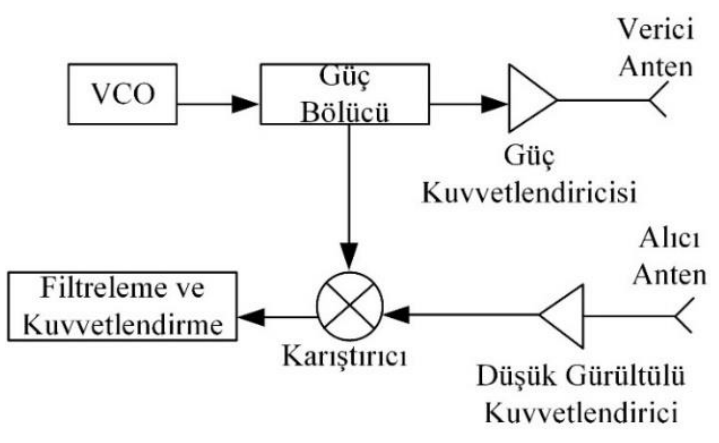

Şekil 1 FMCW radarın temel blok diyagramı 
Voltaj kontrollü osilatörün testere dişi ve üçgen gibi sinyallerle uyarılması sonucu; chirp olarak adlandırılan ve frekansı $\mathrm{f}_{\text {basslanglc }}$ 'dan $\mathrm{f}_{\text {bitiș }}$ 'e kaydırılan bir sinüzoid sinyal üretilir. $\mathrm{FMCW}$ radarın ürettiği sinyalin frekans-zaman ve genlik-zaman grafiği Şekil-2'de görülmektedir.
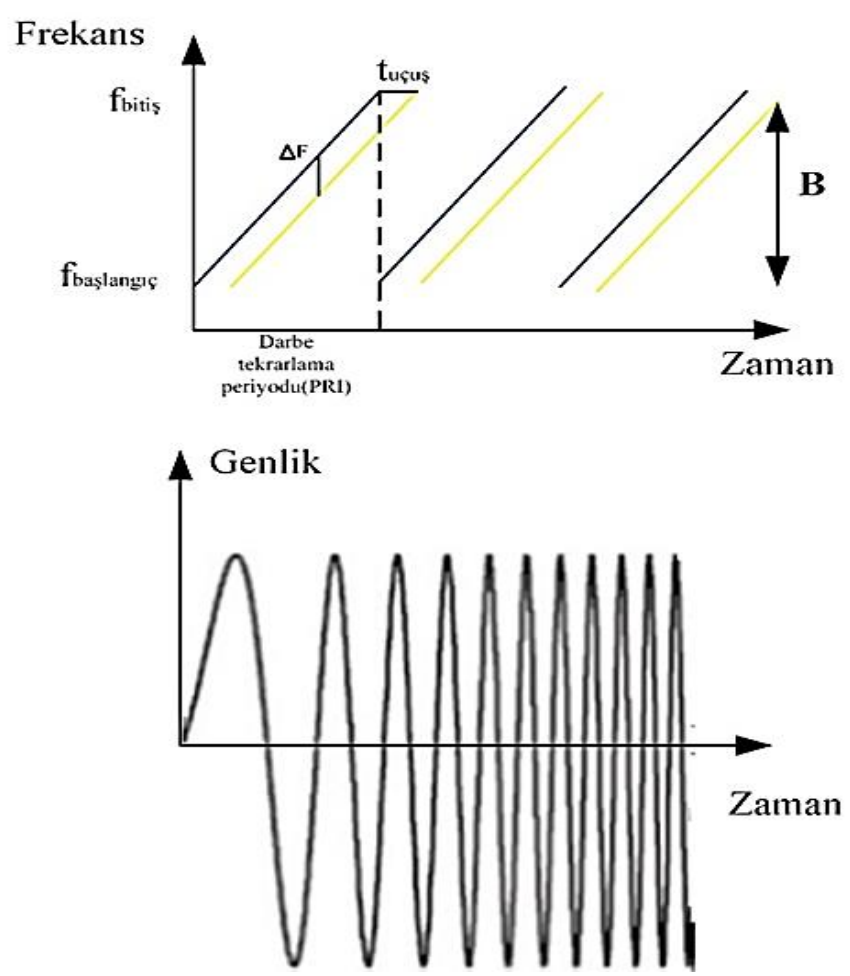

Şekil 2 FMCW radar sinyalinin frekans-zaman ve genlik-zaman grafiği

VCO çıkışındaki sinyalin büyük bir kısmı kuvvetlendirilmek üzere güç kuvvetlendiricisine iletilirken diğer kısmı güç bölücü ile alıcı katına iletilir. Bu sinyal referans sinyali olarak karıştırıcıda kullanılmaktadır. Kuvvetlendirilen sinyal verici anten vasıtasıyla hedefe gönderilmektedir. Gönderilen sinyal;

$$
S_{G}(t)=A_{G} \cos \left(2 \pi f_{c} t+\pi \frac{B}{T_{C}} t^{2}+\emptyset(t)\right)
$$

şeklinde ifade edilir. Burada $A_{G}$ gönderilen sinyalin genliği, $f_{c}$ sinyalin merkez frekansını, $B$ sinyalin bant genişliğini, $T_{c}$ chirp sinyalinin süresini ve $\emptyset(t)$ ise başlangıç fazını göstermektedir. Hedeften yansıyan sinyal radarın alıcı anteni vasıtasıyla alınır. Alınan sinyal;

$$
S_{A}(t)=A_{A} \cos \left(2 \pi f_{c}\left(t-t_{u c ̧ u s ~}\right)+\pi \frac{B}{T_{C}}\left(t-t_{u c ̧ u s ~}\right)^{2}+\emptyset\left(t-t_{u c ̧ u s ~}\right)\right)
$$

şeklinde ifade edilir. $A_{A}$ alınan sinyalin genliğini, $t_{u c u s ~}$ radarın alıcı anteninden $\mathrm{R}(\mathrm{t})=\mathrm{R}_{0}+\mathrm{x}(\mathrm{t})$ mesafesindeki hedefe sinyalin gidiş geliş süresini göstermektedir. Uçuş süresi,

$$
t_{u c ̧ u s ̧}=\frac{2 R(t)}{c}
$$

ile ifade edilir. $c$ ışık hızını göstermektedir. Alıcı antenden alınan sinyal düşük gürültülü kuvvetlendirici ile kuvvetlendirilir. Ardından karıştırıcıda gönderilen sinyalle karıştırılarak düşürülür (down-convert). Elde edilen sinyal orta frekans sinyalidir ve hedef içeriğgi hakkında doğrudan bilgiler veren beat sinyali olarak isimlendirilir. Karıştırıcı çıkışındaki sinyal alçak geçiren filtreden ve kuvvetlendiriciden geçirildikten sonra

$$
S_{B}(t)=A_{B} \cos \left(2 \pi\left[\frac{B}{T_{C}} t_{u c ̧ u s}\right] t+2 \pi f_{c} t_{u c ̧ u s}+\pi \frac{B}{T_{C}} t_{u c ̧ u s}^{2}+\Delta \emptyset(t)\right)
$$

elde edilir. $A_{B}$ sinyal gücünü, zamanla değişen birinci terim vuruş frekansını $\left(f_{b}\right)$, diğer iki terim faz bileşenlerini ve $\Delta \emptyset(t)=\emptyset(t)-$ $\emptyset\left(t-t_{u c ̧ u s ̧}\right)$ rezidual faz gürültüsünü gösterir. Rezidual faz gürültüsü düşük menzilli radar uygulamalarında menzil korelasyon etkisinden dolayı göz ardı edilebilir.

(4) eşitliğinde menzille doğrudan ilişkili olan ve her darbe tekrarlama periyodu için $S_{B}(t)$ sinyaline uygulanan hızlı Fourier dönüşümü (FFT) ile menzil profili belirlenir. $R$ menzilinde yer alan bir hedef için vuruş frekansı,

$$
f_{b}=\frac{B}{T_{C}} t_{u \varsigma ̧ u S}=\frac{2 B R(t)}{c T_{C}}
$$


ifadesinde, $\frac{B}{T_{C}}$ oranı gönderilen sinyallerin eğimi olup chirp hızını ifade etmektedir. Menzil çözünürlüğü radarın iki hedefi birbirinden ayırt edebileceği minimum mesafe olarak tanımlanmaktadır. Bant genişliği $B$ ’nin artmasıyla doğru orantılıdır. Menzil çözünürlüğü,

$$
\Delta R=\frac{c}{2 B}
$$

ile gösterilmektedir.

$$
\emptyset_{b}=2 \pi f_{c} t_{u c ̧ u \varsigma}+\pi \frac{B}{T_{C}} t_{u c ̧ u s}^{2}
$$

(7) eşitliğinde $\pi \frac{B}{T_{C}} t_{u c ̧ u s ~}^{2}$ ifadesi sıfıra çok yakın olduğundan ihmal edilir. Dolayısıyla faz,

$$
\emptyset_{b}(\mathrm{r})=\frac{4 \pi f_{c} R(\mathrm{r})}{c}
$$

şeklinde ifade edilir. (8) menzil geçmişi $R(\mathrm{r})$ olan hedef için beat sinyaline karşılık gelen yavaş zamanın fazını göstermektedir. Böylece her menzil çözünürlüğü ifadesi için (menzil adımı) hayati sinyallerin belirlenmesinde $R(\mathrm{r})$ geçmişinin belirlenmesi solunum ve kalp atış oranlarının hesaplanmasına sebep olmaktadır.

Tablo 1. Simülasyon için FMCW Radar Parametreleri

\begin{tabular}{c|c}
\hline Parametre & Dĕ̌ er \\
\hline Merkez frekansı $\left(f_{c}\right)$ & $24.125 \mathrm{GHz}$ \\
\hline Bant genişliği $(B)$ & $250 \mathrm{MHz}$ \\
\hline Darbe Tekrarlama Periyodu $\left(T_{c}\right)$ & $5 \mathrm{~ms}$ \\
\hline Tutarlı $\dot{I}_{\text {Şleme Aralığ }(C P I)}(C) \mathrm{sn}$ \\
\hline Örnekleme frekansı $\left(f_{s}\right)$ & $120 \mathrm{kHz}$ \\
\hline
\end{tabular}

Tablo 2. Simülasyon için hedef parametreleri

\begin{tabular}{c|c|c}
\hline Parametre & Hedef 1 & Hedef 2 \\
\hline Menzil & $5 \mathrm{~m}$ & $8 \mathrm{~m}$ \\
\hline Solunum hareketi & $1 \mathrm{~cm}$ & $1 \mathrm{~cm}$ \\
\hline Solunum frekansı & $0.4 \mathrm{~Hz}$ & $0.3 \mathrm{~Hz}$ \\
\hline Kalp Atış hareketi & $1 \mathrm{~mm}$ & $1 \mathrm{~mm}$ \\
\hline Kalp Atış frekansl & $1.3 \mathrm{~Hz}$ & $1.2 \mathrm{~Hz}$ \\
\hline
\end{tabular}

Kullanılan FMCW radarın alıcı kısmı dördün (quadrature) yapıya sahiptir. Bu yapı gelen sinyali birbirinden $90^{\circ}$ faz farklı iki sinyale dönüştürür. Bu sinyaller sirası ile $I$ ve $Q$ sinyalleri olarak isimlendirilir.

Hayati sinyallerin eldesi için kullanılan sinyal işleme prosedürü şu şekildedir:

- I ve Q için elde edilen $\boldsymbol{S}_{\boldsymbol{B}}(\boldsymbol{t})$ vuruş sinyalleri $(\mathrm{I}+\mathrm{jQ})$ birleştirilerek kompleks ham veri matrisi $\mathrm{S}_{\mathrm{Hм}}[\mathrm{N}, \mathrm{M}] \mathrm{N}$ satır ve $\mathrm{M}$ sütundan oluşturulur.

- Radar sinyal işlemede matrisin satılları ve sütunları yavaş zaman ve hızlı zaman eksenine karşılık gelir. Satıllar boyunca (hızlı zaman ekseninde) $\mathrm{S}_{\text {нм}}[\mathrm{N}, \mathrm{M}]$ 'nin FFT'si alınarak menzil profili elde edilir.

- Menzil profili matrisinde menzil kutucuklarındaki zirveler hedeflerin bulunduğu menzillere karşllık gelir. İlgili menzildeki faz değişimi hayati sinyallerle ilişkilendirilir.

- Menzil profilinde hedeflerin bulunduğu sinyaller alınarak faz açısı bulunur $\emptyset_{\boldsymbol{b}}(\boldsymbol{n})$ ve faz açma (unwrapping) işlemi uygulanarak $\widehat{\emptyset}_{\boldsymbol{b}}(\boldsymbol{n})$ elde edilir.

- Ardından menzil-yer değiş̧irme geçmişi $\boldsymbol{R}[\boldsymbol{n}]=\frac{\boldsymbol{c} \widehat{\phi}_{\boldsymbol{b}}(\boldsymbol{n})}{\boldsymbol{4 \pi f _ { c } \boldsymbol { c }}}$ şeklinde hesap edilir. Son olarak hayati sinyalleri elde etmek için Doppler FFT uygulanır. Her hedefin Doppler FFT'si hayati sinyallerin frekans bileşenlerinin tespit edilmesini sağlamaktadır.

\section{Araştırma Sonuçları}

Tablo-2'de belirlenen hedef parametrelerine göre oluşturulan matrisin satırları boyunca uygulanan FFT sonucu elde edilen menzil profili Şekil-3'de gösterilmektedir. FMCW radarın menzil çözünürlüğü 0.6 metredir. 9. menzil kutusunda (4.8-5.4 m) 5 metredeki hedef ve benzer şekilde 8 metrede bulunan hedef ise 14.menzil kutusunda (7.8-8.4 m) gösterilmektedir. 5 metredeki hedef tespiti hatasız tespit edilirken $8 \mathrm{~m}$ mesafede olan hedef $8.125 \mathrm{~m}$ ile $\% 1.56$ hata ile bulunmuştur. 
Menzil profilinde hedeflerin bulunduğu sinyallerin sinyal işleme kısmında bahsedildiği gibi faz açıları tespit edilerek faz açma işlemi uygulanmıştır. Sabit iki hedef için göğüs kafesi hareketinden elde edilen yer değiştirme geçmişi Şekil-4'de gösterilmektedir. Gögüs duvar hareketi kaynaklı yer değiştirme geçmişi elde edilen hedefler için hayati sinyallerin tespiti Doppler FFT uygulanarak gerçekleştiril-mektedir. Elde edilen hayati sinyallerin frekans bileşenleri Şekil-5’te gösterilmektedir. Hedef 1 ve hedef 2 için göğüs duvarı hareketine bağı elde edilen solunum sayısı \%2.29 hata oranı ile tespit edilmiştir. Benzer sonuçlar kalp atışı sayısı için aynı hata oranları ile elde edilmiştir.

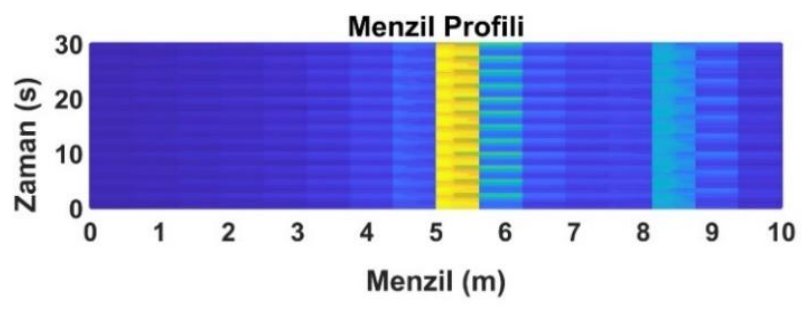

Şekil 3 Hedef Parametrelerine göre Elde Edilen Menzil Profili

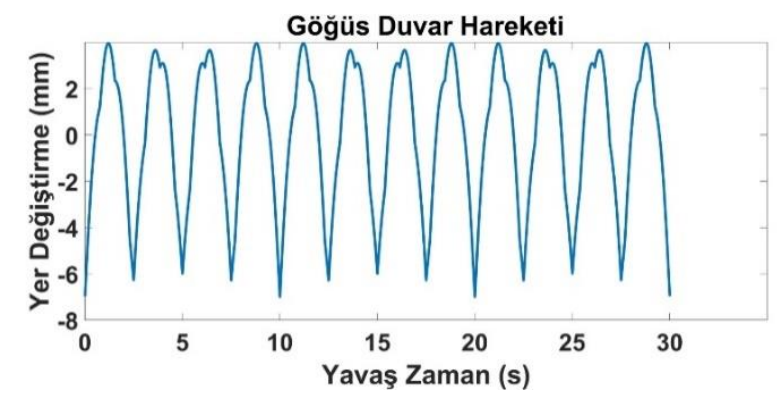

(a)

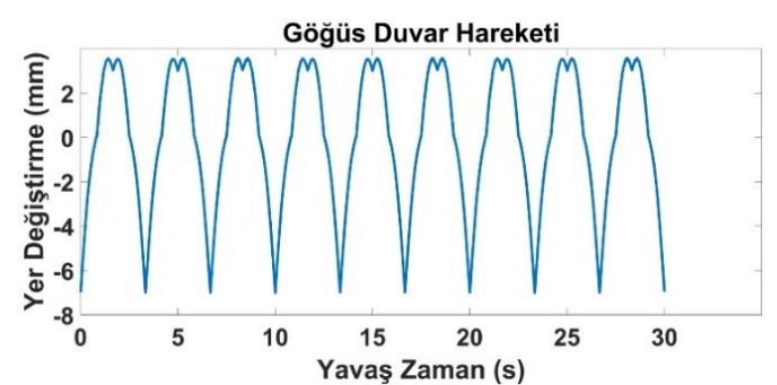

(b)

Şekil 4 Göğüs kafesi hareketinden elde edilen yer değiştirme geçmişi. (a) Hedef 1, (b) Hedef 2

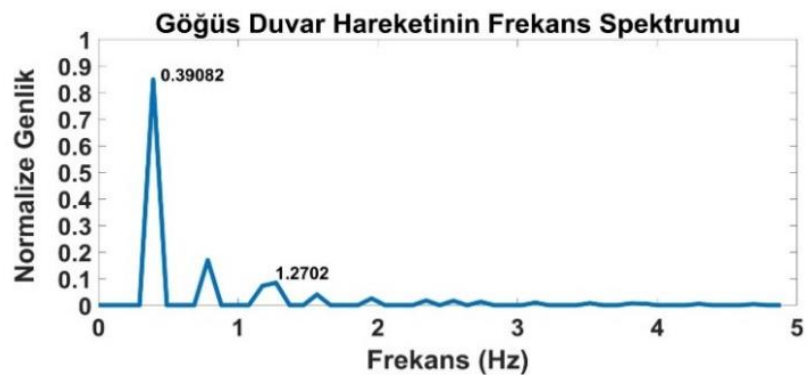

(a)

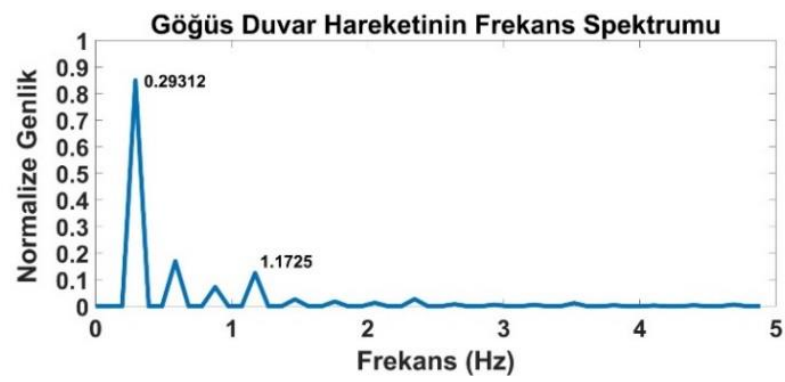

(b)

Şekil 5 Göğüs hareketinden elde edilen hayati sinyallerin frekans bileşenleri. (a) Hedef 1, (b) Hedef 2.

\section{Sonuç}

Bu çalışmada mevcut radarlar hakkında kısa bilgiler ve frekans modüleli sürekli dalga radarın genel yapısı ve avantajları sunulmaktadır. FMCW radar için bir sinyal işleme algoritması ile çoklu insan hedefi için hayati sinyallerin elde edilmesi simüle edilerek sağlanmıştır. 5 metre ve 8 metrede bulunan iki hedef için menziller sırasıyla $\% 0$ ve $\% 1.56$ hata oranıyla tespit edilmiştir. Her iki hedef için solunum ve kalp atışı hata oranları \%2.29 olarak hesaplanmıştır. Bu sonuçlara göre bu radar tipi hem menzil tespitinde hem de hayati sinyallerin eldesi düşünüldüğünde tıbbi uygulamalarda başarılı sonuçlar elde edecektir. Gelecekte mevcut çalışmanın pratiğe dönüştürülmesi amaçlanmaktadır.

\section{Kaynakça}

Muñoz-Ferreras, J. M., Wang, J., Peng, Z., Li, C., \& Gómez-García, R. (2019, May). Fmcw-radar-based vital-sign monitoring of multiple patients. In 2019 IEEE MTT-S International Microwave Biomedical Conference (IMBioC) (Vol. 1, pp. 1-3). IEEE.

Andersen, N., Granhaug, K., Michaelsen, J. A., Bagga, S., Hjortland, H. A., Knutsen, M. R., ... \& Wisland, D. T. (2017). A 118-mw pulse-based radar soc in 55-nm cmos for non-contact human vital signs detection. IEEE Journal of Solid-State Circuits, 52(12), 3421-3433. 
Alizadeh, M., Shaker, G., De Almeida, J. C. M., Morita, P. P., \& Safavi-Naeini, S. (2019). Remote monitoring of human vital signs using mm-Wave FMCW radar. IEEE Access, 7, 54958-54968.

Anishchenko, L., Zhuravlev, A., \& Chizh, M. (2019). Fall Detection Using Multiple Bioradars and Convolutional Neural Networks. Sensors, 19(24), 5569.

Kagawa, M., Suzumura, K., \& Matsui, T. (2016, August). Sleep stage classification by non-contact vital signs indices using Doppler radar sensors. In 2016 38th Annual International Conference of the IEEE Engineering in Medicine and Biology Society $(E M B C)($ pp. 4913-4916). IEEE.

Li, C., Cummings, J., Lam, J., Graves, E., \& Wu, W. (2009). Radar remote monitoring of vital signs. IEEE Microwave Magazine, 10(1), 47-56.

Tran, V. P., Al-Jumaily, A. A., \& Islam, S. M. S. (2019). Doppler radar-based non-contact health monitoring for obstructive sleep apnea diagnosis: A comprehensive review. Big Data and Cognitive Computing, 3(1), 3.

Lin, F., Zhuang, Y., Song, C., Wang, A., Li, Y., Gu, C., ... \& Xu, W. (2016). SleepSense: A noncontact and cost-effective sleep monitoring system. IEEE transactions on biomedical circuits and systems, 11(1), 189-202.

Adib, F., Mao, H., Kabelac, Z., Katabi, D., \& Miller, R. C. (2015, April). Smart homes that monitor breathing and heart rate. In Proceedings of the 33rd annual ACM conference on human factors in computing systems (pp. 837-846).

Hu, W., Zhao, Z., Wang, Y., Zhang, H., \& Lin, F. (2013). Noncontact accurate measurement of cardiopulmonary activity using a compact quadrature Doppler radar sensor. IEEE Transactions on Biomedical Engineering, 61(3), 725-735.

Tu, J., Hwang, T., \& Lin, J. (2016). Respiration rate measurement under 1-D body motion using single continuous-wave Doppler radar vital sign detection system. IEEE Transactions on Microwave Theory and Techniques, 64(6), 1937-1946.

Seflek, I., Acar, Y. E., \& Yaldiz, E. (2020). Small Motion Detection and Non-Contact Vital Signs Monitoring with Continuous Wave Doppler Radars. Elektronika ir Elektrotechnika, 26(3), 54-60.

He, M., Nian, Y., \& Gong, Y. (2017). Novel signal processing method for vital sign monitoring using FMCW radar. Biomedical Signal Processing and Control, 33, 335-345.

Lee, H., Kim, B. H., Park, J. K., Kim, S. W., \& Yook, J. G. (2019). A resolution enhancement technique for remote monitoring of the vital signs of multiple subjects using a $24 \mathrm{GHz}$ bandwidth-limited FMCW radar. IEEE Access, 8, 1240-1248.

Ahmad, A., Roh, J. C., Wang, D., \& Dubey, A. (2018, April). Vital signs monitoring of multiple people using a FMCW millimeterwave sensor. In 2018 IEEE Radar Conference (RadarConf18) (pp. 1450-1455). IEEE.

Mercuri, M., Lorato, I. R., Liu, Y. H., Wieringa, F., Van Hoof, C., \& Torfs, T. (2019). Vital-sign monitoring and spatial tracking of multiple people using a contactless radar-based sensor. Nature Electronics, 2(6), 252-262. 